\begin{abstract}
THE granulocyte colony-stimulating factor (G-CSF) plays an important role in normal granulopoiesis. Its functions are mediated by specific receptors on the surface of responsive cells and, upon ligand binding, several cytoplasmic tyrosine kinases are activated. The cytoplasmic region proximal to the membrane of the G-CSF receptor (G-CSF-R) transduces proliferative and survival signals, whereas the distal carboxy-terminal region transduces maturation signals and suppresses the receptor's proliferative signals. Mutations in the G-CSF-R gene resulting in truncation of the carboxy-terminal region have been detected in a subset of patients with severe congenital neutropenia who developed acute myelogenous leukemia (AML). In addition, the AML1-ETO fusion protein, expressed in leukemic cells harboring the $t(8 ; 21)$, disrupt the physiological function of transcription factors such as $\mathrm{C} / \mathrm{EBP} \alpha$ and $\mathrm{C} / \mathrm{EBP}$, which in turn deregulate G-CSF-R expression. The resulting high levels of G-CSF-R and G-CSF-dependent cell proliferation may be associated with pathogenesis of AML with $t(8 ; 21)$. Moreover, in vitro and in vivo studies demonstrated that G-CSF may act as a co-stimulus augmenting the response of PML-RAR $\alpha$ acute promyelocytic leukemia cells to all-transretinoic acid treatment. Finally, in the PLZF-RAR $\alpha$ acute promyelocytic leukemia transgenic model, G-CSF deficiency suppressed leukemia development. Altogether, these data suggest that the G-CSF signaling pathway may play a role in leukemogenesis.
\end{abstract}

Key words: Granulocyte-colony stimulating factor, Acute myelogenous leukemia, Leukemogenesis

\section{Granulocyte colony-stimulating factor and leukemogenesis}

\author{
Lorena Lobo de Figueiredo, Rodrigo Siqueira de \\ Abreu e Lima and Eduardo Magalhães Rego ${ }^{\text {CA }}$
}

Division of Hematology, Department of Internal Medicine, Medical School of Ribeirão Preto, University of São Paulo, Av. Bandeirantes 3900, Campus USP, 14049-900, Ribeirão Preto, São Paulo, Brazil

\author{
${ }^{\mathrm{CA}}$ Corresponding Author \\ Tel: + 55166022610 \\ Fax: + 55166336695 \\ E-mail: emrego@hcrp.fmrp.usp.br
}

\section{Introduction}

The term acute myelogenous leukemia (AML) refers to a group of neoplastic disorders characterized by the transformation of immature progenitors of the bone marrow, which exhibit morphological and immunophenotypic features common to the myeloid lineage. An important characteristic common to all subtypes of AML is the block of differentiation. Genetically, AML results from acquired mutations, frequently associated with chromosomal translocations, resulting in the generation of chimeric genes and fusion proteins. ${ }^{1}$ In most of the fusion genes associated with AML pathogenesis, one of the involved genes encodes for a transcription factor, which is physiologically involved in hematopoietic differentiation. In other cases, in the absence of chromosomal translocations, the transcription factors themselves are mutated. Therefore, the most common genetic mechanism that is associated with AML is the aberrant expression of a transcription factor or the production of an abnormal hybrid transcription factor. ${ }^{2}$

The granulocyte colony-stimulating factor (G-CSF) is a cytokine secreted by the stromal cells of the hematopoietic organs that influence the survival, proliferation and differentiation of myeloid progenitors. ${ }^{3}$ The responsiveness of AML cells to G-CSF has been reported extensively. ${ }^{4}$ The spontaneous in vitro proliferation of AML blasts and their growth response greatly vary and do not depend on the number or affinity of the growth factor. Particularly, AML cells harboring the $\mathrm{t}(15 ; 17)$ or the $\mathrm{t}(8 ; 21)$ may differentiate upon G-CSF administration. ${ }^{5-8}$ Moreover, mutations on the G-CSF receptor (G-CSF-R) detected in patients with severe congenital neutropenia (SCN) who developed AML suggest that the deregulation of this pathway may contribute to leukemogenesis. ${ }^{9-11}$ Finally, recent data demonstrated that transgenic mice expressing the $t(11 ; 17) /$ acute promyelocytic leukemia (APL)-associated fusion protein PLZFRAR $\alpha$ depend on G-CSF stimulus in order to develop 
full-blown leukemia. This review discusses the current state of knowledge regarding the role of G-CSF in leukemogenesis.

\section{Molecular mechanisms of G-CSF action}

In vitro low concentrations of G-CSF stimulate the formation of granulocyte colonies exclusively, whereas higher concentrations can also stimulate small number of macrophage and mixed macrophage/granulocyte colonies. In addition, G-CSF enhances mature neutrophil function, including their antibody-dependent cellular cytotoxicity and their fMLP-induced superoxide production. ${ }^{12,13}$ Therefore, G-CSF seems to be responsible for both a 'steadystate' and 'emergency-state' neutrophil production. In humans, for example, low neutrophil counts co-exist with high serum G-CSF levels in some clinical situations, suggesting a homeostatic regulatory function for this growth factor. ${ }^{14}$ On the other hand, when extra neutrophils are required acutely, such as during infections, G-CSF concentrations increase dramatically (from less than $100 \mathrm{pg} / \mathrm{ml}$ up to $2000 \mathrm{pg} / \mathrm{ml}$ ), thus acting as an emergency factor by augmenting granulopoiesis. In fact, endotoxins as wells as inflammatory mediators cause an increase of the transcription of the G-CSF gene and of the release of active G-CSF protein. ${ }^{15}$

The biologic functions of G-CSF are mediated by specific receptors on the surface of responsive cells. The G-CSF-R, a single polypeptide containing 813 amino acids, is a member of the cytokine receptor superfamily. G-CSF-R forms homo-oligomeric complexes upon binding of a ligand. Like all members of the cytokine receptor superfamily, G-CSF receptor lacks intrinsic tyrosine kinase activity but can activate cytoplasmic tyrosine kinases. Signal transduction pathways that involve Janus tyrosine kinases (Jak1, Jak2, and Tyk2) and signal transducer and activator of transcription proteins (STAT1, STAT3, and STAT5) are linked to the G-CSF receptor. ${ }^{16,17}$ The cytoplasmic region of the G-CSF-R can be divided in two functional domains: membrane-proximal and membrane-distal — the first of which contains two conserved subdomains named boxes 1 and 2, whereas the membrane distal contains a less conserved box 3 sequence. ${ }^{18}$ In addition, there are four tyrosine (Y) residues at positions 704, 729, 744 and 764 (three of them in the membrane-distal domain), which are rapidly phosphorylated upon G-CSF binding, leading to the formation of potential binding sites for signaling molecules that contain Src homology 2 or phosphotyrosine-binding domains. ${ }^{18}$

Proliferation signals from G-CSF-R are predominantly mediated via Y704 and Y764, with a minor contribution from Y744. ${ }^{19}$ The formation of Grb2/ SHP-2, Gr2/p90 and Grb2/Shc/p140 complexes in response to G-CSF binding is mediated by $\mathrm{Y} 764$, whereas Y704 is a direct docking site for SHP-2. ${ }^{20}$ The proliferative response to G-CSF depends mainly on the STATs pathways, STAT5 being a key mediator, since its inhibition in 32Dc13 cells is able to reverse proliferation without affecting differentiation. $^{21}$

Survival and differentiation signals from G-CSF-R are mainly transduced by $\mathrm{Y} 704$ and $\mathrm{Y} 744$, with a lesser contribution from Y729, and correlates with the capacities of these tyrosines to activate STAT3. However, at high G-CSF concentrations, other survival mechanisms also are activated such as PI3-kinase and mitogen-activated protein kinase. ${ }^{19}$

The most revealing approach to define the in vivo role of hematopoietic growth factors is to study the phenotype of mice genetically deficient in these factors. Murine models of factor deficiency have been reported for monocyte-CSF, granulocytemonocyte (GM)-CSF and G-CSF. ${ }^{22-24}$ The biologic activities of the hematopoietic growth factors G-CSF and GM-CSF overlap significantly both in vitro and after pharmacologic administration in vivo. However, mice deficient in each of these factors have characteristic features. G-CSF-deficient mice have reduced hematopoietic progenitors in the bone marrow and spleen and neutropenia. These animals are markedly predisposed to spontaneous infections, have a reduced long-term survival and a high incidence of reactive type AA amyloidosis. ${ }^{24}$ GMCSF-deficient mice manifest no detectable deficiency in steady-state hematopoiesis, but have increased splenic hematopoietic progenitors and impaired pulmonary surfactant clearance resulting in abnormalities resembling human alveolar proteinosis. They also have a modest impairment of reproductive capacity, a propensity to develop lung and soft tissue infections, and a similarly reduced survival as in GCSF-deficient animals. ${ }^{25}$ These contrasting phenotypes do not exclude the occurrence of additional redundant functions. The residual granulopoiesis in G-CSF-deficient mice, for example, may be attributed to GM-CSF. Moreover, the normal neutrophil level in GM-CSF-deficient animals does not mean that GMCSF has no physiologic function in steady-state granulopoiesis, but indicates that in the presence of regulators such as G-CSF any such role is dispensable. Later studies generated mice lacking both G-CSF and GM-CSF, which demonstrated phenotypic and pathologic features consistent with the additive effects of the constituent genotypes. Thus, these animals manifest a high incidence of soft-tissue and pulmonary infections, and a mortality rate significantly greater than either of the singly deficient strains. Some additional features may also be observed: a greater degree of neutropenia among newborn mice than in those lacking G-CSF alone, 
an increased neonatal mortality rate, and a dominant influence of the lack of G-CSF on splenic hematopoiesis resulting in reduced numbers of splenic progenitors. ${ }^{25}$ These data provide an opportunity to assess the role of these growth factors in normal hematopoiesis and help to understand the consequences of a single factor deficiency.

\section{Acquired G-CSF receptor mutations in SCN}

A number of studies suggest that an abnormal response of granulocytic progenitor cells to G-CSF may be involved in the pathogenesis of SCN. This disorder, also named Kostmann's syndrome, is characterized by persistent neutropenia and bone marrow maturation arrest of neutrophil precursors at the promyelocytic or myelocytic stage. ${ }^{17}$ It occurs as both an autosomal recessive or dominant disorder, but many more sporadic than inherited cases have been reported. Patients, usually children, have severe neutropenia (absolute blood neutrophil count less than $\left.0.2 \times 10^{9} / 1\right)$ and may die from infectious diseases in their early life. ${ }^{10}$ Pharmacologic administration of G-CSF increases neutrophil blood counts and enables long-time survival, but approximately $10-15 \%$ of the patients develop secondary myelodisplastic syndrome or AML. ${ }^{11}$ The fact that precursor cells from most of these patients retain some capacity to proliferate suggests that no defect in maturation, a characteristic of AML, is present in SCN. Thus, it is possible that an impairment in the signaling pathway through the G-CSF receptor may contribute to the pathogenesis of this condition.

Mutations in the G-CSF receptor gene resulting in truncation of the carboxy-terminal region have been detected in a subset of patients with SCN and AML evolving from SCN. ${ }^{9,10,17}$ These mutations, detected in cells of the myeloid lineage only, have been found to localize to a critical cytoplasmic region spanning nucleotides 2384-2429 of the G-CSF receptor, and lead to truncation of the distal cytoplasmic region of the G-CSF receptor, rendering cells hypersensitive to G-CSF. So far, five types of truncation mutations have been found and there has been some controversy over their involvement in the pathogenesis of SCN among the various published studies.

Mitsui et al. ${ }^{17}$ generated transgenic mice (TM) expressing the mutant G-CSF receptor equivalent to truncations at amino acids 718 and 731 in human GCSF receptor, those previously reported to be related to transformation to myelodisplastic syndrome/AML in SCN. Both of the mutant receptor TM had one-third of peripheral neutrophil cell counts compared with wild-type receptor TM, and also showed impaired resistance to the infection with Staphylococcus aureus. Moreover, the bone marrow of these TM had an increased percentage of immature myeloid cells, which is a feature of SCN. Hypersensitivity of myeloid progenitors to stimulation with G-CSF was also observed in the truncated G-CSF TM. These results suggest the involvement of mutations in the G-CSF receptor in the pathogenesis of $\mathrm{SCN}$ and prolonged myeloid cell survival under G-CSF stimulation. This latter effect may induce proliferative stress to myeloid progenitors, and additional oncogenic events could underlie the development of AML in patients with antecedent SCN.

Aprikyan et al. ${ }^{10}$ described cellular and molecular studies of 12 SCN patients, including five patients that evolved to develop AML, that revealed impaired proliferative characteristics and accelerated apoptosis of bone marrow progenitors cells in SCN compared with 11 healthy controls as demonstrated by flow cytometry analysis. This study also revealed heterozygous deletion or substitution mutations in the neutrophil elastase (NE) gene in nine of 12 patients comparing with controls. The authors conclude that the impaired survival of myeloid progenitor cells is probably driven by the expression of mutant NE, which may be the cellular mechanism responsible for neutropenia in SCN. Finally, they suggest that acquired G-CSF mutations may initiate signaling events that override the pro-apoptotic effect of mutant NE in primitive progenitor cells, resulting in an expansion of the abnormal AML clone. This statement is in accordance with Hunter and Avalos, who have showed that mutations in the G-CSF receptor in SCN/AML confer resistance to apoptosis and enhance cell survival, events mediated by activation of PI3-kinase and its downstream targets Akt and Bad, in response to G-CSF. This extension of cell survival would permit the acquisition of additional oncogenic events that could lead to development of AML.

\section{G-CSF in AML with $\mathrm{t}(8 ; 21) / A M L 1-E T O$}

Approximately 5-12\% of AML cases harbor translocations between chromosomes 8 and 21 [t( $8 ; 21)(\mathrm{q} 22$; q22)] that generate the AML1-ETO chimeric gene. ${ }^{26}$ The AML1-ETO fusion protein blocks normal granulocytic differentiation through the disruption of the function of a transcription factor named CCAAT enhancer binding protein $\alpha(\mathrm{C} / \mathrm{EBP} \alpha) .{ }^{27} \mathrm{C} / \mathrm{EBP} \alpha$ function is essential to normal granulocytopoiesis as demonstrated by the fact that the non-conditional inactivation of the $C / E B P \alpha$ gene in mice (knockout) led to the complete absence of mature granulocytes, without affecting other lineages, including monocytes. Conversely, the enforced expression of $\mathrm{C} / \mathrm{EBP} \alpha$ in bipotential myeloid progenitors resulted in induction of granulocytic differentiation and inhibition of monocytic development. ${ }^{28}$ There are two hypothetical models of the mechanism through 
which AML1-ETO can disrupt normal C/EBP $\alpha$ function: (a) AML1-ETO fusion protein can recognize and bind to the AML1 binding site on DNA and recruit co-repressors resulting in the inhibition of the physiological activation of promoters of granulocytic specific genes by C/EBP $\alpha$; or (b) AML1-ETO can inhibit a positive feedback loop exerted by C/ $\mathrm{EBP} \alpha$ on its own expression (positive autoregulation) (Fig. 1). ${ }^{2}$ Corroborating with the second hypothesis is the detection of decreased C/EBP $\alpha$ RNA levels in $\mathrm{t}(8 ; 21)$ patients. ${ }^{27}$ Among the target genes of $\mathrm{C} / \mathrm{EBP} \alpha$ are G-CSF-R, IL- $6, E 2 F, C-M Y C$ and defensin. ${ }^{2}$ Interestingly, G-CSF treatment can lead to in vitro and in vivo differentiation of $\mathrm{t}(8,21)$ AML cell samples. Shimizu et al. ${ }^{29}$ showed that AML1-ETO might modulate cell responses to G-CSF since cells transfected with this fusion protein showed increased proliferation in response to G-CSF without differentiating into mature granulocytes. Moreover, the ectopic expression of AML1-ETO was found to induce the expression of G-CSF receptor, an effect that does not seem to depend on the AML1-binding sequence, but on the C/ EBP binding site.

In addition to $\mathrm{C} / \mathrm{EBP} \alpha$, another $\mathrm{C} / \mathrm{EBP}$ isoform that is deregulated by AML1-ETO is the $\mathrm{C} / \mathrm{EBP} \varepsilon$, which is almost exclusively expressed in the granulocytic lineage and preferentially up-regulated during granulocytic differentiation. The expression of C/EBPE was significantly up-regulated in the cells ectopically expressing AML1-ETO (Fig. 1). The overexpression of $\mathrm{C} / \mathrm{EBP} \varepsilon$ induced expression of G-CSF receptor and GCSF-dependent cell proliferation. High levels of GCSF receptor expression might provide leukemia cells a growth advantage, and may also suppress apoptosis. ${ }^{29}$ However, this up-regulation of G-CSF receptor does not completely explain all the effects of AML1-ETO in leukemogenesis. It is possible that additional factors, whose expression would be deregulated by AML1-ETO, are required to fully express its transforming activity.

\section{G-CSF and APL}

APL is a distinct-subtype of AML that corresponds to the M3 and M3 variant of the French American British classification and to AML subtype with $\mathrm{t}(15 ; 17)(\mathrm{q} 22 ; \mathrm{q} 12)$ and variants according to World Health Organization. APL is characterized by: (a) its invariable association with reciprocal and balanced translocations always involving the retinoic receptor $\alpha(R A R \alpha)$ gene locus on chromosome $17 \mathrm{q} 21$; (b) the clonal expansion of myeloid precursors presenting a block of differentiation at the promyelocytic stage; and (c) its unique sensitivity to the differentiating action of the all-trans-retinoic acid (ATRA). At the molecular level, the $R A R \alpha$ gene on chromosome 17 can be fused to the promyelocytic leukaemia gene $(P M L)$, to the promyelocytic leukaemia zinc finger ( $P L Z F)$ gene, to the nucleophosmin (NPM) gene, to the nuclear mitotic apparatus (NuMA) gene, or to the signal transducer and activator of transcription $5 \mathrm{~B}$ (STAT 5B) gene located on chromosome 15, chromosome 11 , chromosome 5 , chromosome 11 , or chromosome 17 , respectively. In the vast majority of APL cases, the $t(15 ; 17) /$ PML-RAR $\alpha$ is involved and ATRA treatment induces long-term remissions. However, the rare APL cases harboring the $\mathrm{t}(11 ; 17) /$ PLZF-RAR $\alpha$

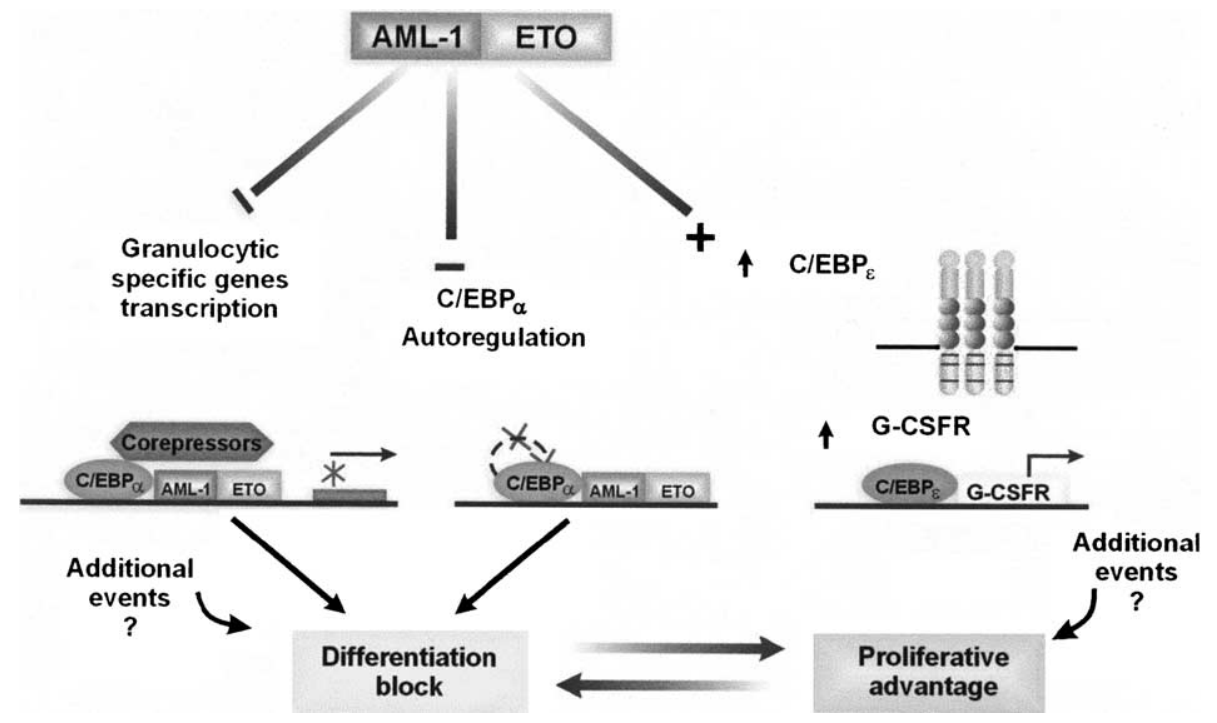

FIG. 1. Hypothetical models of the mechanisms through which AML1-ETO fusion protein disrupt CEBP $\alpha$ function. AML1-ETO can recruit repressor complexes to AML1 binding sites on the promoter regions of granulocytic specific genes and/or inhibit a positive feedback loop exerted by $\mathrm{C} / \mathrm{EBP} \alpha$ on its own promoter. Both mechanisms lead to the decrease of $\mathrm{C} / \mathrm{EBP} \alpha$ transcriptional activity and block of granulocytic differentiation. In addition, AML1-ETO may induce up-regulation of C/EBP $\varepsilon$ which by its turn lead to overexpression of the G-CSF receptor and provide the leukemic cells with proliferative advantage. 
do not respond to ATRA and the prognosis is dismaying. ${ }^{30}$

The expression of the G-CSF-R, as well as the proliferative response induced by G-CSF, is higher in APL cells compared with other AML subtypes. ${ }^{5}$ In vitro studies have shown that induction of differentiation of $P M L-R A R \alpha$-positive cells by ATRA can be enhanced when G-CSF is applied as a co-stimulus. ${ }^{6}$ Cassinat et al. $^{31}$ reported that G-CSF in combination with retinoic acid enhanced the terminal differentiation of fresh t(15;17)-positive APL blasts. Katayama et $a .^{5}$ described the achievement of complete remission with G-CSF treatment in an APL patient resistant to both cytotoxic agents and ATRA. The finding that APL cells are exquisitely responsive to G-CSF supports the view that G-CSF is useful for augmentation of their vulnerability to cell-cycle specific agents. ${ }^{5}$

Jansen et al. $^{32}$ studied a patient with a $\mathrm{t}(11 ; 17)$ positive APL and determined that the combined use of ATRA and G-CSF could overcome the maturation block of the leukemic cells. With retinoic acid and GCSF treatment alone, complete granulocytic maturation of the leukemic cells occurred in vivo, followed by a complete cytogenetical and hematological remission. Bone marrow and blood became negative in fluorescence in situ hybridization analysis, and semi-quantitative polymerase chain reaction showed a profound reduction of $P L Z F-R A R \alpha$. The response was transient, as fluorescence in situ hybridizationpositive cells reappeared in the bone marrow after 7 weeks of treatment. This study suggests that $\mathrm{t}(11 ; 17)$ positive leukemia cells are not intrinsically resistant to retinoic acid and that the G-CSF co-stimulus may be sufficient to render the cells ATRA sensitive.

TM expressing the PML-RAR $\alpha$ fusion gene under the control of the human cathepsin $G$ promoter ( $h C G-P M L-R A R \alpha$ ) develop a form of leukemia that resembles human APL, presenting the characteristic block of myeloid differentiation at the promyelocytic stage and the sensitivity to ATRA treatment. In contrast to leukemia in $h C G-P M L-R A R \alpha$ TM, leukemia in $h C G-P L Z F-R A R \alpha$ TM lacks the distinctive block of differentiation at the promyelocytic stage of myelopoiesis and is characterized by leukocytosis and infiltration of all organs by terminally differentiated myeloid cells (a picture that resembles human chronic myeloid leukemia). In both TM models, leukemia develops after a long latency. Moreover, hCG-PLZF-RAR $\alpha$ TM do not respond to RA treatment. $^{33}$

Lieschke et al $^{34}$ analyzed the relevance of G-CSF and GM-CSF stimuli for leukemia development in hCG-PLZF-RAR $\alpha$ TM. By crossing these TM with knockout mice for either G-CSF (G-CSF ${ }^{-/}$) or GM$\mathrm{CSF}\left(\mathrm{GM}_{-} \mathrm{CSF}^{-/-}\right)$, mutants deficient in either growth factor were obtained. The survival data suggested that while wild-type $b C G-P L Z F-R A R \alpha$ and GM-
$\mathrm{CSF}^{-/-}$hCG-PLZF-RAR $\alpha$ mice developed the characteristic transgene-induced leukemia, G-CSF${ }^{-/-}$ hCG-PLZF-RAR $\alpha$ mice did not succumb to leukemia and survived indistinguishably from $\mathrm{G}-\mathrm{CSF}^{-1-}$ controls. ${ }^{34}$ This experiment has demonstrated that aberrant endogenous G-CSF signals seem to be essential for modifying the leukemogenic propensity of fusion genes like $P L Z F-R A R \alpha$. This suggests that therapeutic approaches exploring G-CSF as a co-stimulus may be useful for the treatment of some leukemias.

In fact, Lowenberg et al. ${ }^{35}$ recently published a study including 640 adult AML patients who were randomized to receive or not receive G-CSF before and during their first two courses of chemotherapy. Although the remission rates were similar in both groups, increased overall survival, disease-free survival as well as event-free survival were observed in the G-CSF-treated group. The benefit of chemotherapysensitization by G-CSF was particularly evident among the intermediate-risk subset of patients. Unfortunately, G-CSF priming did not improve the outcome among patients with chemotherapy-refractory, unfavorable-risk AML. ${ }^{35}$ Nevertheless, these results encourage the strategy of using G-CSF priming to extinguish subpopulations of leukemic cells relatively insensitive to chemotherapy and reduce the risk of relapse.

\section{Conclusion}

Although the relevance of G-CSF in the regulation of granulopoiesis is well established, and much of the molecular mechanisms underlying its function has been elucidated, only recently scientific evidence has been gathered to suggest that acquired mutations in G-CSF-R, or the disruption of the G-CSF function by leukemogenic fusion proteins, may contribute to leukemogenesis. In addition, therapeutic approaches using G-CSF to mobilize subpopulations of leukemic cells, rendering them sensitive to chemotherapy, may represent an important progress in leukemia treatment.

\section{References}

1. Tenen DG, Hromas R, Licht JD, et al. Transcription factors, normal myeloid development, and leukemia. Blood 1997; 90: 489-519.

2. Tenen DG. Disruption of differentiation in human cancer: AML shows the way. Nat Rev Cancer 2003; 3: 89-101.

3. Bondurant M, Koury M. Origin and development of blood cells. In: Lee GR, Foerster J, Lukens J, Paraskevas F, Greer JP, Rodgers GM, eds. Wintrobe's Clinical Hematology, Baltimore, MD: Williams \& Wilkins, 1999: $145-168$

4. Dombret H. Granulocytic colony-stimulating factors in the management of patients with acute myeloid leukemia. Hematol Cell Ther 1996; 38 $231-240$.

5. Katayama N, Kita K, Kawakami, et al. Granulocyte colony-stimulating factor and its receptor in acute promyelocytic leukemia. Am J Hematol 1998; 58: $31-35$ 
6. Huang F, Zhao HP, Gao XZ, et al. Recombinant human G-CSF and retinoic acid in synergistically inducing granulocyte differentiation of human promyelocytic leukemic cells. Chin Med J 1992; 105: 707-712.

7. Da Silva N, Meyer-Monard S, Menot ML, et al. Functional G-CSF pathways in $\mathrm{t}(8 ; 21)$ leukemic cells allow for differentiation induction and degradation of AML1-ETO. Hematol J 2000; 1: 316-328.

8. Ferrara F, Schiavone EM, Palmieri S, et al. Complete remission induced by G-CSF in a patient with acute myeloid leukemia with $\mathrm{t}(8 ; 21)(\mathrm{q} 22 ; \mathrm{q} 22)$. Hematol J 2003; 4: 218-221.

9. Hunter MG, Avalos BR. Granulocyte colony-stimulating factor receptor mutations in severe congenital neutropenia transforming to acute myelogenous leukemia confer resistance to apoptosis and enhance cell survival. Blood 2000; 95: 2132-2137.

10. Aprikyan AA, Kutyavin T, Stein S, et al. Cellular and molecular abnormalities in severe congenital neutropenia predisposing to leukemia. Exp Hematol 2003; 31: 372-381.

11. Tidow N, Pilz C, Teichmann B, et al. Clinical relevance of point mutations in the cytoplasmic domain of the granulocyte colonystimulating factor receptor gene in patients with severe congenital neutropenia. Blood 1997; 89: 2369-2375.

12. Metcalf D, Nicola NA. Proliferative effects of purified granulocyte colony-stimulating factor (G-CSF) on normal mouse hemopoietic cells. J Cell Physiol 1983; 116: 198-206.

13. Platzer E, Welte K, Gabrilove JL, et al. Biological activities of a human pluripotent hemopoietic colony stimulating factor on normal and leukemic cells. J Exp Med 1985; 162: 1788-1801.

14. Watari K, Asano S, Shirafuji N, et al. Serum granulocyte colonystimulating factor levels in healthy volunteers and patients with various disorders as estimated by enzyme immunoassay. Blood 1989; 73: $117-$ 122.

15. Basu S, Dunn A, Ward A. G-CSF: function and modes of action (Review). Int J Mol Med 2002; 10: 3-10.

16. Fukunaga R, Ishizaka-Ikeda E, Seto Y, et al. Expression cloning of a receptor for murine granulocyte colony-stimulating factor. Cell 1990; 61 $341-350$.

17. Mitsui T, Watanabe S, Taniguchi $Y$, et al. Impaired neutrophil maturation in truncated murine G-CSF receptor-transgenic mice. Blood 2003; 101 $2990-2995$.

18. Fukunaga R, Seto $\mathrm{Y}$, Mizushima $\mathrm{S}$, et al. Three different mRNAs encoding human granulocyte colony-stimulating factor receptor. Proc Natl Acad Sci USA 1990; 87: 8702-8706.

19. Ward AC, Smith L, de Koning JP, et al. Multiple signals mediate proliferation, differentiation, and survival from the granulocyte colonystimulating factor receptor in myeloid 32D cells. J Biol Chem 1999; 274 $14956-14962$.

20. de Koning JP, Schelen AM, Dong F, et al. Specific involvement of tyrosine 764 of human granulocyte colony-stimulating factor receptor in signal transduction mediated by p145/Shc/GRB2 or p90/GRB2 complexes. Blood 1996; 87: 132-140.

21. Ilaria RL, Jr, Hawley RG, van Etten RA. Dominant negative mutants implicate STAT5 in myeloid cell proliferation and neutrophil differentiation. Blood 1999; 93: 4154-4166.

22. Wiktor-Jedrzejczak W, Bartocci A, Ferrante AW, Jr, et al . Total absence of colony-stimulating factor 1 in the macrophage-deficient osteopetrotic (op/op) mouse. Proc Natl Acad Sci USA 1990; 87: 4828-4832.
23. Stanley E, Lieschke GJ, Grail D, et al. Granulocyte/macrophage colonystimulating factor-deficient mice show no major perturbation of hematopoiesis but develop a characteristic pulmonary pathology. Proc Natl Acad Sci USA 1994; 91: 5592-5596.

24. Lieschke GJ, Grail D, Hodgson G, et al. Mice lacking granulocyte colony-stimulating factor have chronic neutropenia, granulocyte and macrophage progenitor cell deficiency, and impaired neutrophil mobilization. Blood 1994; 84: 1737-1746.

25. Seymour JF, Lieschke GJ, Grail D, et al. Mice lacking both granulocyte colony-stimulating factor (CSF) and granulocyte-macrophage CSF have impaired reproductive capacity, perturbed neonatal granulopoiesis, lung disease, amyloidosis, and reduced long-term survival. Blood 1997; 90: 3037-3049.

26. Brunning R, Matutes E, Flandrin G, et al. Acute myeloid leukemia with recurrent genetic abnormalities. In: Jaffe E, Harris N, Stein H, Vardiman J, eds. Pathology and Genetics of Tumors of Haematopoietic and Lymphoid Tissues, Lyon: IARC Press, 2001: 81-87.

27. Pabst T, Mueller BU, Harakawa N, et al. AML1-ETO downregulates the granulocytic differentiation factor C/EBPalpha in $\mathrm{t}(8 ; 21)$ myeloid leukemia. Nat Med 2001; 7: 444-451.

28. Zhang DE, Zhang P, Wang ND, et al. Absense of granulocyte colonystimulating factor signaling and neutrophil development in CCAAT enhancer binding protein alpha-deficient mice. Proc Natl Acad Sci USA 1997; 94: 569-574.

29. Shimizu K, Kitabayashi I, Kamada N, et al. AML1-MTG8 leukemic protein induces the expression of granulocyte colony-stimulating factor (G-CSF) receptor through the up-regulation of CCAAT/enhancer binding protein epsilon. Blood 2000; 96: 288-296.

30. Rego EM, Pandolfi PP. Analysis of the molecular genetics of acute promyelocytic leukemia in mouse models. Semin Hematol 2001; 38: $54-70$.

31. Cassinat B, Balitrand N, Zassadowski F. Combination of ATRA with GCSF or RARalpha agonists enhances differentiation induction of fresh APL cells. Blood 1998; 92: s596a.

32. Jansen JH, de Ridder MC, Geertsma WM, et al. Complete remission of $\mathrm{t}(11 ; 17)$ positive acute promyelocytic leukemia induced by all-trans retinoic acid and granulocyte colony-stimulating factor. Blood 1999; 94 $39-45$.

33. Rego EM, Pandolfi PP. Reciprocal products of chromosomal translocations in human cancer pathogenesis: key players or innocent bystanders? Tends Mol Med 2002; 8: 396-405.

34. Lieschke G, Pandolfi P, Sony V. PLZF-RARalpha transgenic mice lacking G-CSF, but not those lacking GM-CSF, fail to develop acute myeloid leukemia and live a normal lifespan. Blood 2002; 100: s189a.

35. Lowenberg B, van Putten W, Theobald M, et al. Effect of priming with granulocyte colony-stimulating factor on the outcome of chemotherapy for acute myeloid leukemia. N Eng J Med 2003; 349: 743-752.

\section{Received 26 February 2004}

Accepted 12 March 2004 


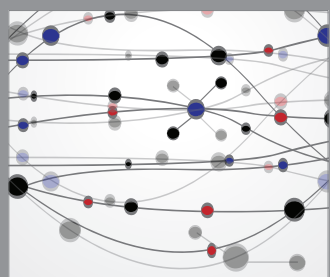

The Scientific World Journal
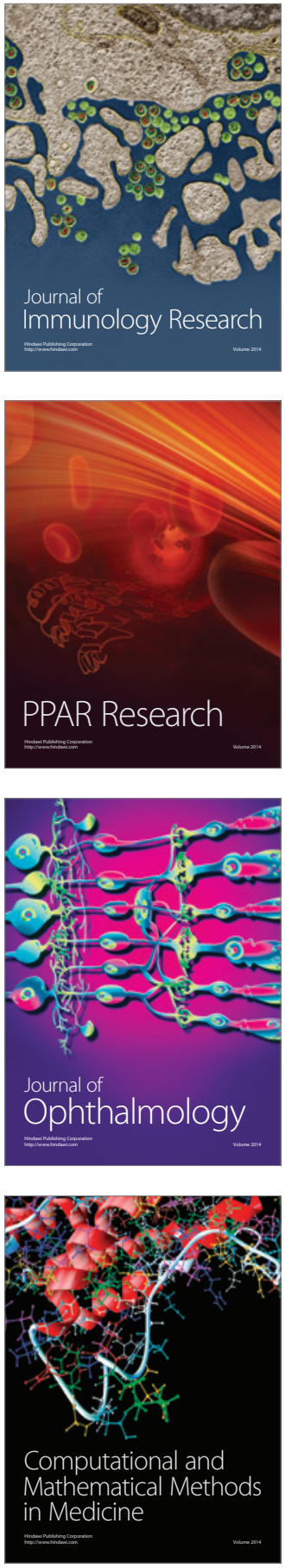

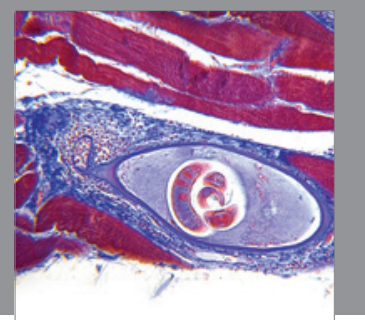

Gastroenterology

Research and Practice
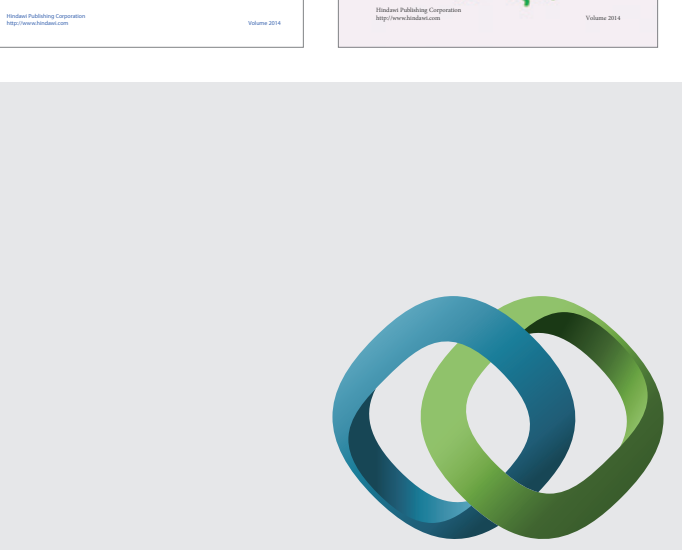

\section{Hindawi}

Submit your manuscripts at

http://www.hindawi.com
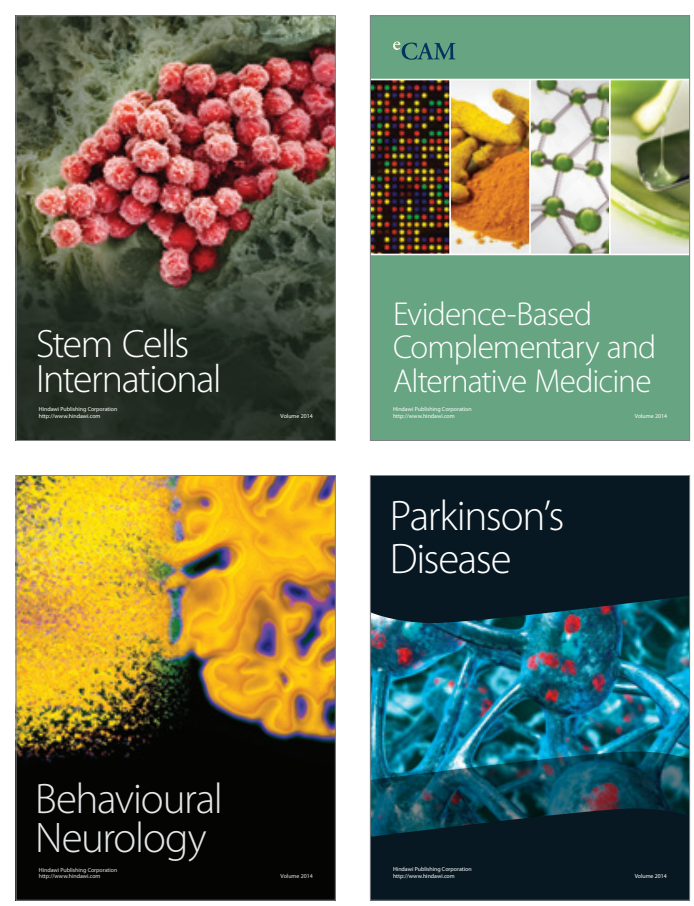

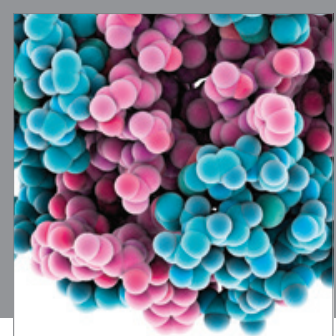

Journal of
Diabetes Research

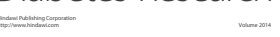

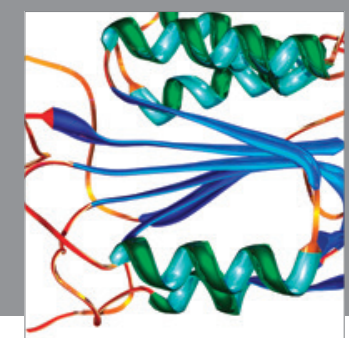

Disease Markers
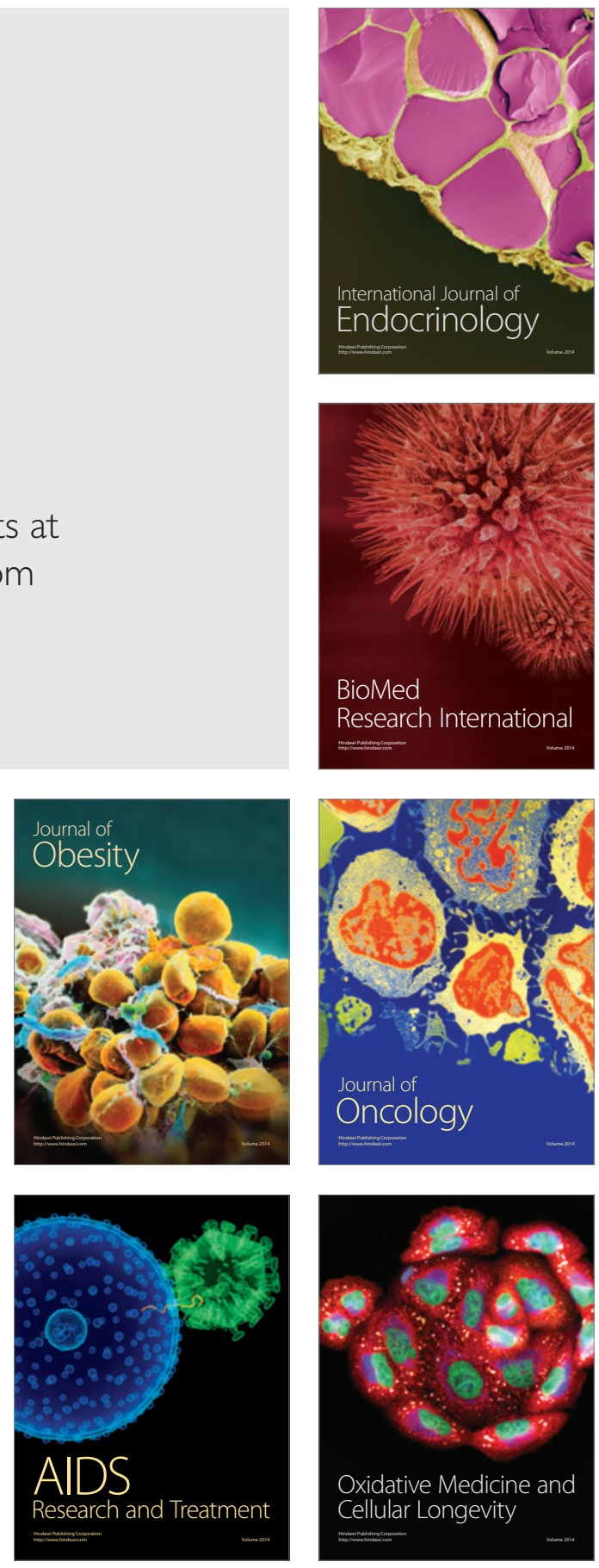